\title{
Influence of microscopic many-body scattering on multi-wavelength VECSEL lasing
}

I. Kilen, J. Hader, S. W. Koch, J. V. Moloney

I. Kilen, J. Hader, S. W. Koch, J. V. Moloney, "Influence of microscopic manybody scattering on multi-wavelength VECSEL lasing," Proc. SPIE 10901, Vertical External Cavity Surface Emitting Lasers (VECSELs) IX, 109010E (4 March 2019); doi: 10.1117/12.2510516

SPIE. Event: SPIE LASE, 2019, San Francisco, California, United States 


\title{
Influence of Microscopic Many-Body Scattering on Multi-Wavelength VECSEL Lasing
}

\author{
I. Kilen ${ }^{a}$, J. Hader ${ }^{a}$, S. W. Koch ${ }^{a, b}$, and J. V. Moloney ${ }^{a, c}$. \\ ${ }^{a}$ College of Optical Sciences, The University of Arizona, \\ 1630 East University Boulevard, Tucson, Arizona 85721, USA; \\ ${ }^{b}$ Department of Physics and Material Sciences Center, Philipps-Universität Marburg, \\ Renthof 5, 35032 Marburg, Germany; \\ ${ }^{c}$ Department of Mathematics, University of Arizona, \\ 617 N. Santa Rita, Tucson, AZ 85721, USA
}

\begin{abstract}
Non-equilibrium multi-wavelength operation of vertical external-cavity surface-emitting lasers (VECSELs) is investigated numerically using a coupled system of Maxwell semiconductor Bloch equations. The propagation of the electromagnetic field is modeled using Maxwell's equations, and the semiconductor Bloch equations simulate the optically active quantum wells. Microscopic many-body carrier-carrier and carrier-phonon scattering are treated at the level of second Born-Markov approximation, polarization dephasing with a characteristic rate, and carrier screening with the static Lindhard formula. At first, an initialization scheme is constructed to study multi-wavelength operation in a time-resolved VECSEL. Intracavity dual-wavelength $\mathrm{THz}$ stabilization is examined using longitudinal modes and an intracavity etalon. In the latter, anti-correlated noise is observed for $\mathrm{THz}$ generation and investigated.
\end{abstract}

Keywords: VECSELs, semiconductor laser, multi-wavelength, simulation, semiconductor Bloch equations

\section{INTRODUCTION}

Vertical external-cavity surface-emitting lasers (VECSELs) are low-cost, versatile lasers that have been used in a wide range of applications from ultrashort mode-locked pulses to high-intensity single- and multi-wavelength output. ${ }^{1-9}$ To date, the highest peak power for continuous wave (CW) operation is $23 \mathrm{~W},{ }^{7}$ while for multiwavelength lasing the peak power has reached $106 \mathrm{~W} .5,6$ The VECSEL has achieved around $100 \mathrm{fs}$ intracavity temporal duration when configured to produce passively mode-locked pulses, ${ }^{8-10}$ with the shortest intracavity pulse duration at $95 \mathrm{fs}^{10}{ }^{10}$ In surface-emitting lasers, the field is not propagating parallel with the quantum well $(\mathrm{QW})$ planes, which implies that a high carrier density is required in the inverted QWs to obtain spectral amplification of the intracavity field. High mirror reflectivity will, in turn, result in the buildup of strong intracavity fields that significantly distort the QW carriers. These conditions enable the VECSEL to be an ideal place to study non-equilibrium many-body dynamics where QW carriers are driven far from quasi-equilibrium Fermi distributions.

In VECSELs, a dual-wavelength output with THz spacing has been generated using both: two mode-locked pulses centered with THz-spacing and with intracavity difference frequency generation (DFG). ${ }^{11,12}$ Stable dualwavelength operation of VECSELs has many applications ranging from spectroscopy, ${ }^{13,14}$ geological and atmospheric measurements using LIDAR, ${ }^{15,16}$ semiconductor laser parameter measurement, ${ }^{17,}{ }^{18} \mathrm{THz}$-imaging, ${ }^{19-22}$ and THz-signal generation. ${ }^{23,24}$

In general, stable dual-wavelength operation in semiconductor lasers has been a popular research topic both experimentally and theoretically. ${ }^{12,25-36}$ Experiments have been able to produce stable dual-wavelength operation, ${ }^{12,25-30}$ however attention has recently been brought to anti-correlated intensity noise in dual-wavelength

Further author information:

I. K.: Electronic mail: ikilen@math.arizona.edu

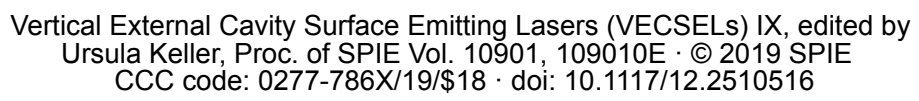

Proc. of SPIE Vol. 10901 109010E-1 
output. $^{29,30}$ Theoretically, rate equations have been the primary tool for analysis of multi-mode operation. Moustafa Ahmed and Minoru Yamada coupled multi-mode rate equations to Langevin noise and characterized regimes of stability of continuous wave and multi-mode operation according to the injection current and linewidth enhancement factor. ${ }^{33}$ Yacomotti et al. experimentally and theoretically observed strong anti-correlated mode dynamics, which maintained near constant total output intensity. ${ }^{34}$ They conclude that the dominant cause of the phenomena was four-wave mixing of the intracavity field.

When utilizing rate equations, the microscopic polarization dynamics is adiabatically eliminated, and carrier densities directly model the influence of optically active QWs. ${ }^{31-35}$ This approach assumes carriers are in quasiequilibrium Fermi distributions and is insensitive to details of ultrafast microscopic carrier dynamics present in VECSEL systems. Microscopic analysis of dual-wavelength operation in semiconductors examined a simple micro-resonator under dual-wavelength operation. ${ }^{36}$ Bäumner et al. used the Maxwell semiconductor Bloch equations (MSBE) at the Hartree-Fock level with rate approximations for carrier scattering and found that an accurate model of multi-wavelength operation requires the inclusion of non-equilibrium carrier dynamics.

In this paper, we will examine dual-wavelength operation in VECSELs and numerically solve the MSBE with carrier scattering computed on the level of the second Born-Markov approximation. ${ }^{37,38}$ In past articles, we have used this model to systematically analyze the role of microscopic dynamics in the mode-locked operation of VECSELs. ${ }^{39-42}$ In contrast to stable mode-locked pulse operation, a VECSEL configured for dual-wavelength output generates an intracavity field that continuously extracts carriers from the gain chip. Hot carriers are replenished in the QWs by an optical pump and scattered to lower energies by carrier-carrier and carrier-phonon scattering. In multi-mode operation, the QW carrier dynamics is also influenced by sum- and difference-frequency generation and does not reach a stable equilibrium. These influences necessitate that the high-dimensional carrier scattering integrals have to be solved at all times, which becomes a significant computational problem.

\section{THEORETICAL BACKGROUND}

A numerical model of a VECSEL consists of coupling Maxwell's equations, for the light field propagation, to the semiconductor Bloch equations that model the optically active QWs. In general for a realistic VECSEL design, these are coupled nonlinear integro differential equations with thousands of variables that are solved until an asymptotic solution is found. Thus before attempting a solution, we will simplify the model to focus on interesting microscopic aspects. In a VECSEL, the light field propagates close to perpendicular relative to the QW planes, and thus we simplify Maxwell's equations to only consider TE-polarized light, $E(z, t)$, propagation along the z-axis according to,

$$
\left[\frac{\partial^{2}}{\partial z^{2}}-\frac{n(z)^{2}}{c_{0}^{2}} \frac{\partial^{2}}{\partial t^{2}}\right] E(z, t)=\mu_{0} \frac{\partial^{2}}{\partial t^{2}} P(z, t),
$$

where $P(z, t)$ is the macroscopic polarization from the QWs, $c_{0}$ is the speed of light in vacuum, $\mu_{0}$ is the vacuum permeability, and $n(z)$ is the background refractive index. In this situation, we are modeling a linear laser cavity where the gain chip with inverted QWs is placed on the left, while a frequency independent output coupling mirror is placed on the right after an air gap. The gain chip is build up from a distributed Bragg reflector (DBR) with ten inverted QWs modeled by the SBE, a cap layer, and an anti-reflection coating. At each material layer interface, the propagating light field will experience transmission and reflection and an additional macroscopic polarization when interacting with QWs.

The VECSEL contains multiple inverted QWs that have independent microscopic dynamics simulated using the SBE. ${ }^{37}$ For each QW, the dynamic variables are the electron (hole) occupation numbers, $n_{\lambda(\nu), \mathbf{k}}^{\mathrm{e}(\mathrm{h})}$, and the microscopic polarizations, $p_{\lambda, \nu, \mathbf{k}}$, discretized by the crystal momentum, k. For electrons (holes) in conduction (valence) bands $\lambda(\nu)$, the microscopic many-body dynamics is governed by,

$$
\begin{aligned}
\frac{\partial}{\partial t} p_{\lambda, \nu, \mathbf{k}} & =-\frac{\mathrm{i}}{\hbar} \sum_{\lambda_{1}, \nu_{1}}\left(e_{\lambda, \lambda_{1}, \mathbf{k}}^{\mathrm{e}} \delta_{\nu, \nu_{1}}+e_{\nu, \nu_{1}, \mathbf{k}}^{\mathrm{h}} \delta_{\lambda, \lambda_{1}}\right) p_{\lambda_{1}, \nu_{1}, \mathbf{k}}-\mathrm{i}\left(n_{\lambda, \mathbf{k}}^{\mathrm{e}}+n_{\nu, \mathbf{k}}^{\mathrm{h}}-1\right) \Omega_{\lambda, \nu, \mathbf{k}}+\Lambda_{\lambda, \nu, \mathrm{spont}}^{p}+\left.\frac{\partial}{\partial t} p_{\lambda, \nu, \mathbf{k}}\right|_{\text {corr }}, \\
\frac{\partial}{\partial t} n_{\lambda(\nu), \mathbf{k}}^{\mathrm{e}(\mathrm{h})} & =-2 \operatorname{Im}\left(\Omega_{\lambda, \nu, \mathbf{k}}\left(p_{\lambda, \nu, \mathbf{k}}\right)^{*}\right)+\Gamma_{\lambda(\nu), \mathrm{scatt}}^{\mathrm{e}(\mathrm{h})}+\Lambda_{\lambda, \nu, \mathrm{spont}}^{n}+\left.\frac{\partial}{\partial t} n_{\lambda(\nu), \mathbf{k}}^{e(h)}\right|_{\text {corr }} .
\end{aligned}
$$


This couples to Eq. 1 through the macroscopic polarization that can be computed by summing over the microscopic polarizations, $P(z, t)=\sum_{\mathbf{k}} d_{\mathbf{k}}^{\lambda, \nu} p_{\lambda, \nu, \mathbf{k}}$, where $d_{\mathbf{k}}^{\lambda, \nu}$ is the dipole matrix element. The coulomb renormalized single particle energies, $e_{\lambda, \lambda_{1}, \mathbf{k}}^{\mathrm{e}}$ and $e_{\nu, \nu_{1}, \mathbf{k}}^{\mathrm{h}}$, and the effective Rabi frequency, $\Omega_{\lambda, \nu, \mathbf{k}}$, at the Hartree-Fock level are computed with the Coulomb potential, $V_{|\mathbf{k}-\mathbf{q}|}^{\lambda, \nu_{1}, \nu, \lambda_{1}}$, from

$$
\begin{aligned}
& e_{\lambda, \lambda_{1}, \mathbf{k}}^{\mathrm{e}}=\epsilon_{\lambda, \mathbf{k}}^{\mathrm{e}} \delta_{\lambda, \lambda_{1}}-\sum_{\lambda_{2}, \mathbf{q}} V_{|\mathbf{k}-\mathbf{q}|}^{\lambda, \lambda_{2}, \lambda_{1}, \lambda_{2}} n_{\lambda_{2}, \mathbf{q}}^{\mathrm{e}} \\
& e_{\nu, \nu_{1}, \mathbf{k}}^{\mathrm{h}}=\epsilon_{\nu, \mathbf{k}}^{\mathrm{h}} \delta_{\nu, \nu_{1}}-\sum_{\nu_{2}, \mathbf{q}} V_{|\mathbf{k}-\mathbf{q}|}^{\nu, \nu_{2}, \nu_{1}, \nu_{2}} n_{\nu_{2}, \mathbf{q}}^{\mathrm{h}}
\end{aligned}
$$

and

$$
\Omega_{\lambda, \nu, \mathbf{k}}=\omega_{\mathrm{R}}+\frac{1}{\hbar} \sum_{\lambda_{1}, \nu_{1}, \mathbf{q} \neq \mathbf{k}} V_{|\mathbf{k}-\mathbf{q}|}^{\lambda, \nu_{1}, \nu, \lambda_{1}} p_{\lambda_{1}, \nu_{1}, \mathbf{q}} .
$$

Where, $\omega_{\mathrm{R}}=d_{\mathbf{k}}^{\lambda, \nu} E(z, t) / \hbar$, is the Rabi frequency, and $\epsilon_{\lambda(\nu), \mathbf{k}}^{\mathrm{e}(\mathrm{h})}$ is the electron (hole) single particle energy. In Eq. 2, the optical barrier pumping, $\Gamma_{\lambda(\nu) \text {,scatt }}^{\mathrm{e}(\mathrm{h})}$, the spontaneous emission with $\Lambda_{\lambda, \nu \text {,spont }}^{n}$, the polarization dephasing, $\left.\frac{\partial}{\partial t} p_{\lambda, \nu, \mathbf{k}}\right|_{\text {corr }}$, the carrier-carrier and carrier-phonon scattering, $\left.\frac{\partial}{\partial t} n_{\lambda(\nu), \mathbf{k}}^{e(h)}\right|_{\text {corr }}$, and carrier screening are terms are discussed further below. In general, the polarization dephasing and carrier scattering are multi-dimensional integrals over the microscopic polarization and carrier occupation numbers. ${ }^{38}$

The carrier scattering, $\left.\frac{\partial}{\partial t} n_{\lambda(\nu), \mathbf{k}}^{e(h)}\right|_{\text {corr }}$, at the level of second Born-Markov is given by the sum of carrier-carrier and carrier-phonon scattering. These terms and more details has been explored in detail by Hader et al., ${ }^{38}$ and are listed here for reference. First, the carrier scattering integrals are expressed by,

$$
\begin{aligned}
\left.\frac{d}{d t} n_{\lambda, \mathbf{k}}^{\mathrm{e}}\right|_{\mathrm{cc}}= & \sum_{\substack{\lambda_{1}, \lambda_{2}, \lambda_{3} \\
\mathbf{q}, \mathbf{k}^{\prime}}} \hat{V}_{|\mathbf{q}|}^{\lambda, \lambda_{1}, \lambda_{2}, \lambda_{3}}\left(\hat{V}_{|\mathbf{q}|}^{\lambda, \lambda_{1}, \lambda_{2}, \lambda_{3}}-\hat{V}_{\left|\mathbf{k}-\mathbf{k}^{\prime}\right|}^{\lambda, \lambda_{1}, \lambda_{2}, \lambda_{3}}\right) \mathcal{D}\left(\epsilon_{\mathbf{k}}^{\mathrm{e}, \lambda}+\epsilon_{\mathbf{k}^{\prime}-\mathbf{q}}^{\mathrm{e}, \lambda_{1}}-\epsilon_{\mathbf{k}^{\prime}}^{\mathrm{e}, \lambda_{2}}-\epsilon_{\mathbf{k}-\mathbf{q}}^{\mathrm{e}, \lambda_{3}}\right) . \\
& \left(\left(1-n_{\lambda_{2}, \mathbf{k}^{\prime}}^{\mathrm{e}}\right)\left(1-n_{\lambda_{3}, \mathbf{k}-\mathbf{q}}^{\mathrm{e}}\right) n_{\lambda, \mathbf{k}}^{\mathrm{e}} n_{\lambda_{1}, \mathbf{k}^{\prime}-\mathbf{q}}^{\mathrm{e}}-\left(1-n_{\lambda, \mathbf{k}}^{\mathrm{e}}\right)\left(1-n_{\lambda_{1}, \mathbf{k}^{\prime}-\mathbf{q}}^{\mathrm{e}}\right) n_{\lambda_{2}, \mathbf{k}^{\prime}}^{\mathrm{e}} \mathrm{e}_{\lambda_{3}, \mathbf{k}-\mathbf{q}}^{\mathrm{e}}\right) \\
+ & \sum_{\substack{\lambda_{1}, \nu_{1}, \nu_{2} \\
\mathbf{q}, \mathbf{k}^{\prime}}}\left|\hat{V}_{|\mathbf{q}|}^{\lambda, \nu_{1}, \nu_{2}, \lambda_{1}}\right|^{2} \mathcal{D}\left(\epsilon_{\mathbf{k}-\mathbf{q}}^{\mathrm{e}, \lambda_{1}}-\epsilon_{\mathbf{k}}^{\mathrm{e}, \lambda}+\epsilon_{\mathbf{k}^{\prime}+\mathbf{q}}^{\mathrm{h}, \nu_{1}}-\epsilon_{\mathbf{k}^{\prime}}^{\mathrm{h}, \nu_{2}}\right) . \\
& \left(\left(1-n_{\lambda_{1}, \mathbf{k}-\mathbf{q}}^{\mathrm{e}}\right)\left(1-n_{\nu_{1}, \mathbf{k}^{\prime}+\mathbf{q}}^{\mathrm{h}}\right) n_{\lambda, \mathbf{k}}^{\mathrm{e}} n_{\nu_{2}, \mathbf{k}^{\prime}}^{\mathrm{h}}-\left(1-n_{\lambda, \mathbf{k}}^{\mathrm{e}}\right)\left(1-n_{\nu_{2}, \mathbf{k}^{\prime}}^{\mathrm{h}}\right) n_{\lambda_{1}, \mathbf{k}-\mathbf{q}}^{\mathrm{e}} n_{\nu_{1}, \mathbf{k}^{\prime}+\mathbf{q}}^{\mathrm{h}}\right) .
\end{aligned}
$$

where $\hat{V}_{|\mathbf{q}|}^{\lambda, \nu_{1}, \nu_{2}, \lambda_{1}}$ is the screened Coulomb matrix element, and $\mathcal{D}(E)$ is an energy conserving delta function. In general, these expressions cannot be analytically evaluated and requires numerical quadratures. Secondly, the phonon scattering is defined by specifying the lattice parameters: the LO-phonon energy $\left(\hbar \omega_{L O}=33.95 \mathrm{meV}\right)$, lattice temperature $(300 \mathrm{~K})$, and the dielectric constants at high- $\left(\epsilon_{\infty}=11.24\right)$ and low- $(\epsilon=13.47)$ frequencies. These values are determined from interpolation of table values. ${ }^{43}$ Now, we can express the carrier-phonon contribution as:

$$
\begin{aligned}
\left.\frac{d}{d t} n_{\lambda, \mathbf{k}}^{\mathrm{e}}\right|_{\mathrm{cp}} & =\sum_{\lambda_{1}, \mathbf{q}}\left|g_{\mathbf{q}}^{\lambda \lambda_{1}}\right|^{2}\left\{\mathcal{D}\left(\epsilon_{\mathbf{k}}^{\mathrm{e}, \lambda}-\epsilon_{\mathbf{k}+\mathbf{q}}^{\mathrm{e}, \lambda_{1}}+\hbar \omega_{\mathbf{q}}\right)\left(s_{\mathbf{q}} n_{\mathbf{k}}^{\mathrm{e}, \lambda}\left(1-n_{\mathbf{k}+\mathbf{q}}^{\mathrm{e}, \lambda_{1}}\right)-\left(1+s_{\mathbf{q}}\right) n_{\mathbf{k}+\mathbf{q}}^{\mathrm{e}, \lambda_{1}}\left(1-n_{\mathbf{k}}^{\mathrm{e}, \lambda}\right)\right)\right. \\
& \left.-\mathcal{D}\left(\epsilon_{\lambda_{1}, \mathbf{k}-\mathbf{q}}^{\mathrm{e}}-\epsilon_{\lambda, \mathbf{k}}^{\mathrm{e}}+\hbar \omega_{\mathbf{q}}\right)\left(s_{\mathbf{q}} n_{\lambda_{1}, \mathbf{k}-\mathbf{q}}^{\mathrm{e}}\left(1-n_{\lambda, \mathbf{k}}^{\mathrm{e}}\right)-\left(1+s_{\mathbf{q}}\right) n_{\lambda, \mathbf{k}}^{\mathrm{e}}\left(1-n_{\lambda_{1}, \mathbf{k}-\mathbf{q}}^{\mathrm{e}}\right)\right)\right\}
\end{aligned}
$$

Here, $g_{\mathbf{q}}^{\lambda \lambda_{1}}$ is the Fröhlich matrix element, ${ }^{44,45} s_{\mathbf{q}}$ is the phonon distribution, and $\hbar \omega_{\mathbf{q}}$ is the LO-phonon energy.

Optical pumping injects hot carriers at higher momentum values and the propagating electromagnetic field extracts carriers from the lower momentum states. During VECSEL operation, carrier scattering will cool down carrier energies by transporting the carriers to lower momentum states. In particular, carrier-phonon scattering will relax carriers towards the lattice temperature, where energy is primarily extracted around LO-phonon energy 
resonances. Simultaneously, carrier-carrier scattering will drive carriers towards quasi-Fermi distributions at the instantaneous energy and temperature.

A further simplification is to model two parabolic energy bands separated by a bandgap, $E_{\mathrm{g}}$. In this case the transition energy is given by, $\hbar \omega_{\mathbf{k}}=E_{\mathrm{g}}+\frac{\hbar^{2} \mathbf{k}^{2}}{2 m_{\mathrm{e}}}+\frac{\hbar^{2} \mathbf{k}^{2}}{2 m_{\mathrm{h}}}$, where $m_{\mathrm{e}(\mathrm{h})}$ is the effective electron (hole) mass. Assuming a high QW carrier density, such as during VECSEL operation, we express the polarization dephasing as a characteristic timescale, $\left.\frac{\partial}{\partial t} p_{\lambda, \nu, \mathbf{k}}\right|_{\text {corr }}=-\tau_{d e p h} p_{\lambda, \nu, \mathbf{k}}$, and the screening using the static Lindhard formula. ${ }^{37}$ The characteristic time for the optical barrier pumping of electrons (holes) is given by, $\tau_{\text {scatt }}=30 \mathrm{ps,}$ and is modeled as a relaxation to a constant background Fermi distribution, $f_{\lambda(\nu), \mathbf{k}}^{\mathrm{e}(\mathrm{h})}$, at the lattice temperature $(300 \mathrm{~K})$ and a density $3 \cdot 10^{16} \mathrm{~m}^{-2}$. The pumping term is given by, $\Gamma_{\lambda(\nu), \text { scatt }}^{\mathrm{e}(\mathrm{h})}=-\left(n_{\lambda(\nu), \mathbf{k}}^{\mathrm{e}(\mathrm{h})}-f_{\lambda(\nu), \mathbf{k}}^{\mathrm{e}}\right) / \tau_{\text {scatt }}$. The MSBE model is semiclassical, which means we will have to add spontaneous emission. In a VECSEl, spontaneous emission injects a small noise in the cavity, which can build up in a VECSEL to become a significant output signal. We will use the same model as Bäumner et al., ${ }^{36}$ where we set, $\Lambda_{\lambda, \nu \text {,spont }}^{n}=-\Lambda_{\mathbf{k}}^{\text {spont }} n_{\lambda, \mathbf{k}}^{e} n_{\nu, \mathbf{k}}^{h}$, and, $\Lambda_{\lambda, \nu \text {,spont }}^{p}=\beta \Lambda_{\mathbf{k}}^{\text {spont }} n_{\lambda, \mathbf{k}}^{e} n_{\nu, \mathbf{k}}^{h}$, with a random complex number, $\beta$. The norm of, $\beta$, represents the coupling of spontaneous emission into the cavity and is for simplicity assumed to be unity.

We will consider a linear VECSEL cavity that consists of a gain chip, an air gap, and a frequency independent output coupling mirror. The gain chip is constructed with an AlGaAs DBR, ten inverted $8 \mathrm{~nm}$ wide InGaAs QWs situated in a single anti-node of the DBR stranding wave, a cap layer, and two AR coatings. The gain chip design was also used in ${ }^{39,40}$ to study mode-locked pulses, but pulsed considerations such as dispersion compensating coating are less critical when considering dual-wavelength output. The peak gain has been centered on the DBR stopband at $1030 \mathrm{~nm}$.

\section{RESULTS}

Dual-wavelength operation of a VECSEL starts with spontaneous emission building up energy inside the laser cavity. The intracavity field will experience amplification from the gain chip and loss from the output coupling mirror during a round trip. After thousands of round trips, the intracavity field will become strong enough to distort the gain chip QW carriers away from equilibrium. Thus carriers are no longer in quasi-Fermi equilibrium distributions, which will correspondingly cause the gain chip amplification to become distorted. Next, a period of equilibration begins where the intracavity spectrum reshapes to seek a balance between round trip amplification and cavity loss. Finally, the stable states that the system can settle into are either a stable dual-wavelength operation with two spectral peaks of equal intensity, dual-wavelength output where the two spectral peaks have unequal intensity, or a single wavelength solution where one of the two wavelengths has become significantly suppressed.

A numerical solution of Eqs. (1)-(2) with carrier scattering from Eqs. (5)-(6) is computationally demanding because we are looking for asymptotic solutions. The VECSEL cavity contains ten independent QWs, where the SBE dynamics govern the carriers in each QW. For each QW, integration of the SBE require a sub-femtosecond resolution to resolve the microscopic dynamics, and carrier scattering integrals have to be evaluated at every timestep. Finally, the asymptotic stable state of the intracavity field is obtained after thousands of cavity round trips. To facilitate research on these equations, solvers take advantage of modern shared memory computers and a hybrid MPI-OpenMP scheme is used to evaluate the microscopic scattering integrals in parallel for all QWs. Simulations in this article are run on an SGI UV 2000 shared memory computer with almost 400 cores and 4 TB of memory. The computational problem is still significant and the estimated time to establish the stability of a dual-wavelength simulation is one or more days.

\subsection{Estimation of non-equilibrium amplification}

Stable dual-wavelength operation traditionally requires that the intracavity amplification is equal to the constant output coupling mirror loss. Thus, a natural start would be to attempt to parametrize the spectral amplification of the gain chip. However, the coupled Eqs. (1)-(2) are nonlinear and the parameter space involves hundreds of continuous and discrete variables such as QW density, cavity field spectral shape and intensity, number of (and placement) of gain chip QWs, the material composition of gain chip, etc. In general, the QW carriers will control the spectral amplification from the gain chip. During dual-wavelength lasing the intracavity field will 
extract low momentum carriers and the optical pump will inject higher momentum carriers. Simultaneously, carrier scattering will attempt to cool down the carriers by driving them towards quasi-Fermi distributions at the lattice temperature. These dynamic many-body effects will distort the spectral amplification of the gain chip over many thousands of round trips while stable dual-wavelength operation gradually is established.

A condition for stable dual-wavelength operation is that both wavelengths experience equal spectral amplification during a round trip of the laser. To examine this, we approximate the final intracavity field an ideal dual-wavelength signal with frequencies $\left(\omega_{a}, \omega_{b}\right)$ and amplitude, $E_{\mathrm{in}}$. The non-equilibrium amplification of the input signal during lasing conditions is estimated by injecting a dual-wavelength light field of the form, $E(t)=E_{\text {in }}\left(\mathrm{e}^{-\mathrm{i} \omega_{a} t}+\mathrm{e}^{-\mathrm{i} \omega_{b} t}\right)$, on the gain chip until the QW carrier densities stabilize. At this point, we can compute the amplification of each spectral peak by comparing the ideal input to the reflected signal from the gain chip. Here, we do not allow the reflected signal to feed back into the input signal.

The non-equilibrium amplification for any two frequencies $\left(\omega_{a}, \omega_{b}\right)$ can now be determined by scanning over multiple input field amplitudes, of the ideal signal, to determine which (if any) will result in both wavelengths having equal amplification. Fig. 1 shows an example of this scan. The spectral amplification at $\omega_{a}\left(\omega_{b}\right)$ is shown in blue (red) in Fig. 1a). The amplification for both wavelengths has a clear intersection near the intensity $2.5 \mathrm{MW} / \mathrm{cm}^{2}$. As a result, for these two wavelengths and the given field intensity, the gain chip will amplify both wavelengths by $0.69 \%$. In Fig. $1 \mathrm{~b}$ ) a sample output spectra (blue) produced with the above method is compared to the ideal input spectra (red). The spectra are computed after the gain chip QW carriers have stabilized and it is clear that, beyond the amplification of two dominant modes, the output spectra contains multiple low amplitude peaks that are generated by nonlinear wave mixing in the gain chip QWs.
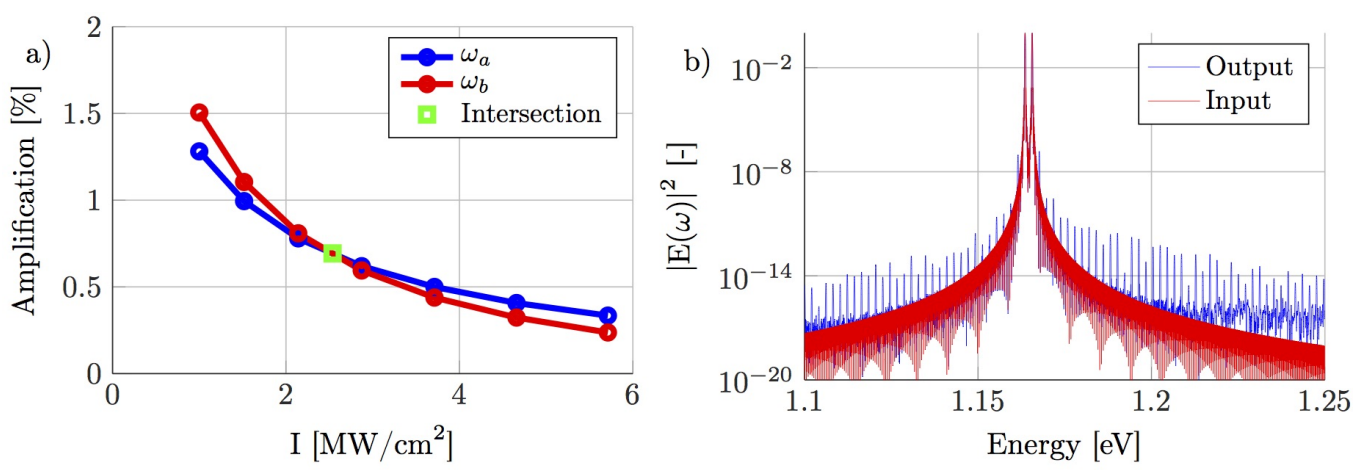

Figure 1: An example of asymptotic output amplification for an ideal dual-wavelength input signal. a) The spectral amplification of both input frequencies as a function of input intensity. b) A sample output spectra (blue) compared to the ideal input (red).

The asymptotic information about the non-equilibrium amplification obtained with the above method can now be used to initialize the dual-wavelength VECSEL close to the final state. Following the above method, an initial ideal input field with frequencies $\left(\omega_{a}, \omega_{b}\right)$ and amplitude, $E_{\text {in }}$, is used to drive the QW carriers close to the desired non-equilibrium state where both frequencies experience equal spectral amplification. Filling up the external air cavity in the process. At this point, we start the self-consistent solution by adding an output coupling mirror with an appropriate loss that balances the amplification from the gain chip. The VECSEL intracavity field is now allowed to evolve from the ideal initial guess to a fully self-consistent solution. From Fig. 1b), it is clear that the initial guess will be reshaped by the feedback and nonlinear influences such as four-wave mixing, phase changes between the two peaks, spectral broadening, etc. Thus a period of equilibration from the initial guess to the final dual-wavelength state is expected.

\subsection{Self-consistent simulations}

In the following examples, we will utilize the previously described method to generate an initial guess of the final non-equilibrium dual-wavelength solution where both frequencies ideally experience equal amplification. To control the output field spectra, one usually has to add additional intracavity filtering. One possibility is to use the longitudinal cavity modes to dictate the output spectrum. Two wavelengths with terahertz separation can be 
selected by using a very short external air cavity, which will result in cavity lengths around tens of micrometer. A second method involves inserting a glass etalon (refractive index 1.5) into a long (about $3.2 \mathrm{~mm}$ ) external air cavity. The longer external cavity results in a denser set of longitudinal cavity modes and the etalon will help to resonantly amplify or dampen modes.

\subsubsection{Longitudinal modes}

To use the previously described initialization method we first find the intracavity field intensity that results in equal dual-wavelength amplification of both spectral peaks. The scan indicates that for energies $\hbar\left(\omega_{a}, \omega_{b}\right)=$ $(1.161 \mathrm{eV}, 1.246 \mathrm{eV})$ we get that a peak intensity $0.5749 \mathrm{MW} / \mathrm{cm}^{2}$ produces equal amplification for both spectral peaks. A short external cavity allows only a few longitudinal modes inside the gain region and specific modes can be selected using the length of the external cavity. Fig. 2 summarizes a stable dual-wavelength simulation that results in a frequency separation of $20 \mathrm{THz}$ filtered with a very short external cavity of length $3.6 \mu \mathrm{m}$.
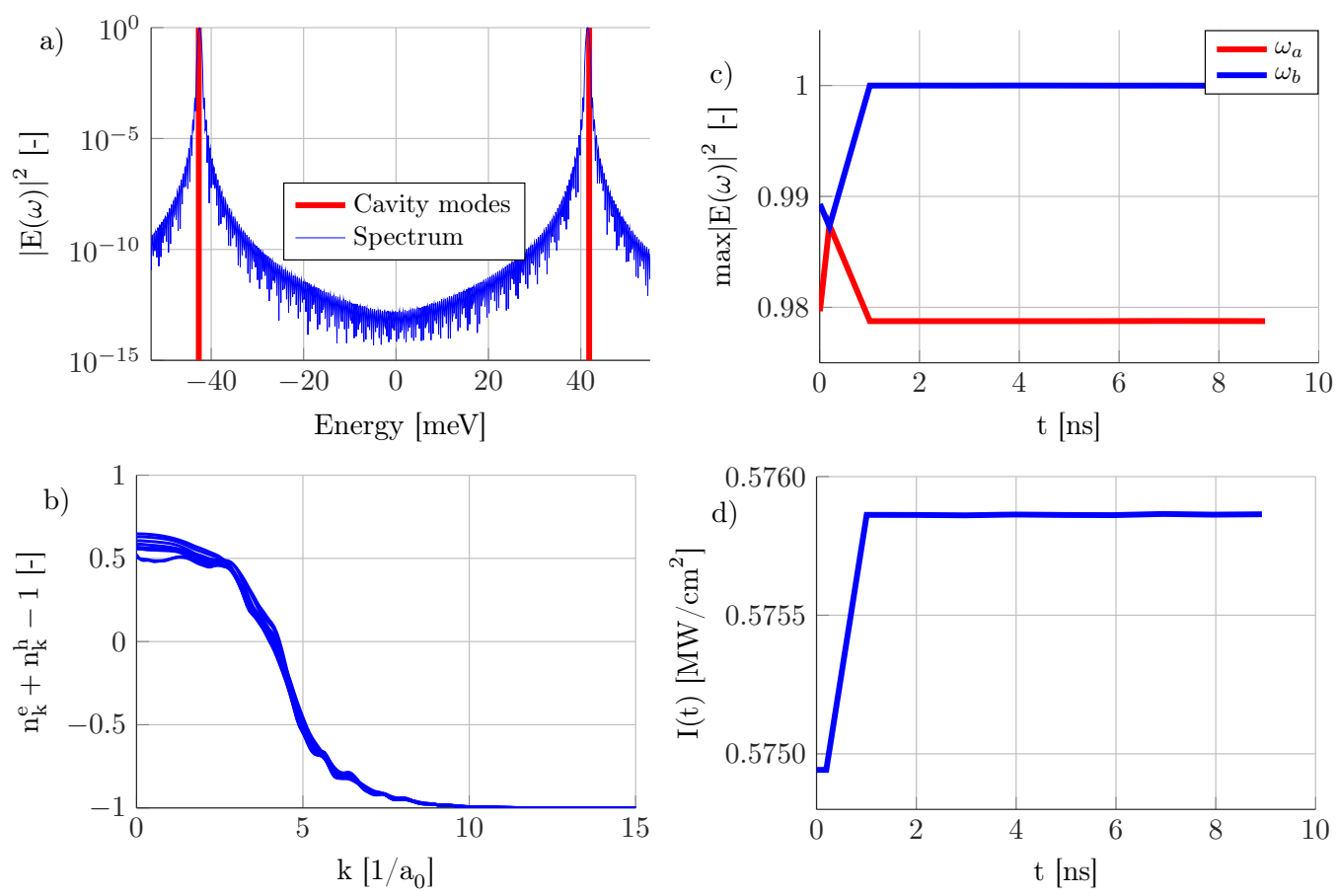

Figure 2: A simulation of dual-wavelength operation from a very short external air cavity of length $3.6 \mu \mathrm{m}$. a) The resulting output spectra, with a frequency separation of $20 \mathrm{THz}$. The energy axis is relative to peak gain. b) A snapshot of each QWs inversion during stable operation. c) The relative amplitude of each spectral peak intensity. d) The total intracavity field intensity.

The output spectra (blue), in Fig. 2a), contains two narrow spectral peaks nearly centered at the predicted cold cavity modes (red vertical bars). A snapshot of each QWs inversion during stable operation is shown in Fig. $2 \mathrm{~b}$ ). The intracavity field is extracting carriers from the low momentum region, but note that there are also multiple distortions near and beyond $k=5$. These distortions correspond to LO-phonon resonances from the carrier-phonon scattering from Eq. (6). In general, the degree of carrier distortion increases with higher instantaneous carrier temperature. In Fig. 2c), the intensity of each spectral peak is monitored over time, and we observe that there is a short stabilization period before the intensities of both frequencies become stationary. This equilibration period is also clear from the total intensity shown in Fig. 2d). The initialization worked as intended for this example, and the data indicates that the desired dual-wavelength output was obtained.

\subsubsection{Intracavity etalon}

Inserting a glass etalon into the external air cavity gives a VECSEL setup that resembles experimental designs that result in dual-wavelength operation. The etalon will create an additional subcavity with a length that can 
be fine-tuned to amplify selected modes resonantly. However, the etalon only offers a coarse frequency selection, and the additional subcavity will inevitably lead to new field dynamics that can destabilize the intracavity field. As an example, we insert a $48.2 \mu \mathrm{m}$ glass $(\mathrm{n}=1.5)$ etalon into the center of a $3.3 \mathrm{~mm}$ external air cavity.
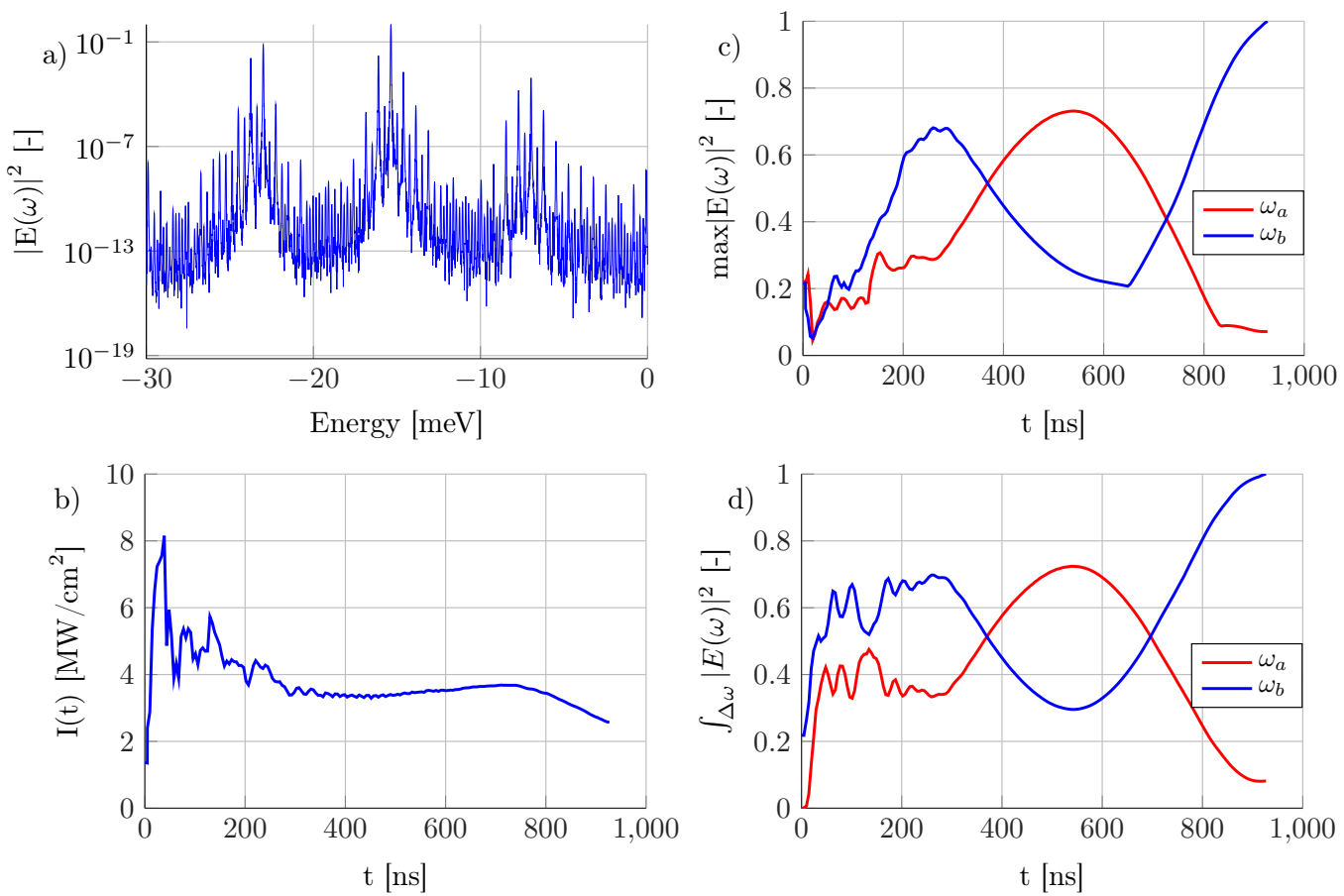

Figure 3: A simulation where an intracavity etalon is centered in a $3.3 \mathrm{~mm}$ external air gap. a) The final output spectra from this simulation with an energy axis relative to peak gain. b) The evolution of the peak intracavity intensity. c) The peak spectral intensity near $\omega_{a}$ and $\omega_{b}$ relative to the maximal intensity at the end of the simulation. d) The integrated spectral intensity in a $\pm 4 \mathrm{meV}$ range around $\omega_{a}$ and $\omega_{b}$ relative to the maximal intensity at the end of the simulation.

Fig. 3a) contains the final output spectra with spectral peaks around $\hbar \omega_{a}=1.180 \mathrm{eV}$ and $\hbar \omega_{b}=1.188 \mathrm{eV}$. The resulting spectrum contains families of modes distributed with about $2 \mathrm{THz}$ spacing. However, it is clear that this is far from the ideal initial guess used during the initialization. A lot of transient dynamics is observed in the peak intensity, shown in Fig. 3b). Indeed, the output spectra never stabilizes during this simulation because of ongoing competition between different spectral peaks. The maximal spectral peak intensity in the two dominant mode families around $\hbar \omega_{a}=1.180 \mathrm{eV}$ and $\hbar \omega_{b}=1.188 \mathrm{eV}$ are shown in Fig. 3c). These spectral peaks have a slow dynamics with occasional changes in derivative, but the individual dominant modes do not appear to be strongly anti-correlated. To analyze this, we will integrate the spectral intensity over each of the two dominant mode families. Fig. 3d) shows that the integrated spectral peak intensities for each mode family are strongly anti-correlated. This anti-correlated intensity noise is also observed in experimental setups. ${ }^{28,30}$ The complex mode dynamics in this example is a result of inserting the etalon into the external air cavity, which introduces subcavities in the VECSEL. The delayed feedback caused by the partial reflections from the propagating intracavity field will eventually feed back to the gain chip QWs. In general, delayed feedback of multimode operation has been known to produce complex dynamics ranging from chaotic, quasi-periodic, to stable operation in lasers. ${ }^{46}$

\section{SUMMARY}

Simulation of dual-wavelength operation in a VECSEL with carrier-carrier and carrier-phonon scattering on the level of second Born-Markov has found examples of stable and anti-correlated multimode dynamics. An initialization method was used to reduce the calculation time by starting the simulations close to the asymptotic 
solution. For a very short cavity the method produced excellent results with sufficiently good spectral filtering. During stable dual-wavelength operation of the VECSEL, the carriers are not in equilibrium Fermi distributions, but the occupation numbers are distorted by carrier-phonon scattering as well as the intracavity lasing field. Anti-correlated intensity noise is observed when including an intracavity etalon in a longer external cavity. In further studies, we will examine the stabilization and origin of the anti-correlated noise.

\section{ACKNOWLEDGEMENTS}

This material is based upon work supported by the Air Force Office of Scientific Research under award number FA9550-17-1-0246.

\section{REFERENCES}

[1] Keller, U. and Tropper, A. C., "Passively modelocked surface-emitting semiconductor lasers," Phys. Rep. 429, 67-120 (2006).

[2] Tropper, A. C., Quarterman, A. H., and Wilcox, K. G., "Ultrafast vertical-external-cavity surface-emitting semiconductor lasers," Advances in Semiconductor Lasers 86, 269-300 (2012).

[3] Tilma, B. W., Mangold, M., Zaugg, C. A., Link, S. M., Waldburger, D., Klenner, A., Mayer, A. S., Gini, E., Golling, M., and Keller, U., "Recent advances in ultrafast semiconductor disk lasers," Light: Science $\mathcal{E}^{\prime}$ Applications 4(7), e310 (2015).

[4] Rahimi-Iman, A., "Recent advances in vecsels," Journal of Optics 18(9), 093003 (2016).

[5] Heinen, B., Wang, T.-L., Sparenberg, M., Weber, A., Kunert, B., Hader, J., Koch, S. W., Moloney, J. V., Koch, M., and Stolz, W., "106 W continuous-wave output power from vertical-external-cavity surfaceemitting laser," Electron. Lett. 48(9), 516 (2012).

[6] Wang, T.-L., Heinen, B., Hader, J., Dineen, C., Sparenberg, M., Weber, A., Kunert, B., Koch, S. W., Moloney, J. V., Koch, M., and Stolz, W., "Quantum design strategy pushes high-power vertical-externalcavity surface-emitting lasers beyond $100 \mathrm{~W}, "$ Laser Photon. Rev. 6(5), L12-L14 (2012).

[7] Zhang, F., Heinen, B., Wichmann, M., Möller, C., Kunert, B., Rahimi-Iman, A., Stolz, W., and Koch, M., "A 23-watt single-frequency vertical-external-cavity surface-emitting laser," Optics express 22(11), 12817-12822 (2014).

[8] Waldburger, D., Link, S. M., Mangold, M., Alfieri, C. G. E., Gini, E., Golling, M., Tilma, B. W., and Keller, U., "High-power 100 fs semiconductor disk lasers," Optica 3(8), 844-852 (2016).

[9] Klopp, P., Griebner, U., Zorn, M., and Weyers, M., "Pulse repetition rate up to $92 \mathrm{GHz}$ or pulse duration shorter than 110 fs from a mode-locked semiconductor disk laser," Appl. Phys. Lett. 98, 071103 (2011).

[10] Laurain, A., Kilen, I., Hader, J., Ruiz Perez, A., Ludewig, P., Stolz, W., Addamane, S., Balakrishnan, G., Koch, S. W., and Moloney, J. V., "Modeling and experimental realization of modelocked vecsel producing high power sub-100 fs pulses," Applied Physics Letters 113(12), 121113 (2018).

[11] Scheller, M., Baker, C. W., Koch, S. W., and Moloney, J. V., "Dual-wavelength passively mode-locked semiconductor disk laser," IEEE Photonics Technology Letters 28(12), 1325-1327.

[12] Scheller, M., Yarborough, J. M., Moloney, J. V., Fallahi, M., Koch, M., and Koch, S. W., "Room temperature continuous wave milliwatt terahertz source," Optics express 18(26), 27112-27117 (2010).

[13] Axner, O., Lejon, M., Magnusson, I., Rubinsztein-Dunlop, H., and Sjöström, S., "Detection of traces in semiconductor materials by two-color laser-enhanced ionization spectroscopy in flames," Applied optics 26(17), 3521-3525 (1987).

[14] Zhou, J., Park, N., Dawson, J. W., Vahala, K. J., Newkirk, M. A., and Miller, B. I., "Terahertz four-wave mixing spectroscopy for study of ultrafast dynamics in a semiconductor optical amplifier," Applied physics letters 63(9), 1179-1181 (1993).

[15] Langbein, J. O., Burford, R. O., and Slater, L. E., "Variations in fault slip and strain accumulation at parkfield, california: Initial results using two-color geodimeter measurements, 1984-1988," Journal of Geophysical Research: Solid Earth 95(B3), 2533-2552 (1990).

[16] Koch, G. J., Barnes, B. W., Petros, M., Beyon, J. Y., Amzajerdian, F., Yu, J., Davis, R. E., Ismail, S., Vay, S., Kavaya, M. J., et al., "Coherent differential absorption lidar measurements of co 2," Applied optics 43(26), 5092-5099 (2004). 
[17] Jiang, S. and Dagenais, M., "Parameter extraction in semiconductor lasers using nearly degenerate four-wave mixing measurements," in [Lasers and Electro-Optics Society Annual Meeting, 1993. LEOS'93 Conference Proceedings. IEEE], 578-579, IEEE (1993).

[18] Liu, J.-M. and Simpson, T. B., "Four-wave mixing and optical modulation in a semiconductor laser," IEEE journal of quantum electronics 30(4), 957-965 (1994).

[19] Kleine-Ostmann, T., Knobloch, P., Koch, M., Hoffmann, S., Breede, M., Hofmann, M., Hein, G., Pierz, K., Sperling, M., and Donhuijsen, K., "Continuous-wave thz imaging," Electronics Letters 37(24), 1461-1463 (2001).

[20] Hu, B. B. and Nuss, M. C., "Imaging with terahertz waves," Optics letters 20(16), 1716-1718 (1995).

[21] Yamaguchi, S., Fukushi, Y., Kubota, O., Itsuji, T., Ouchi, T., and Yamamoto, S., "Brain tumor imaging of rat fresh tissue using terahertz spectroscopy," Scientific reports 6, 30124 (2016).

[22] Federici, J. F., Schulkin, B., Huang, F., Gary, D., Barat, R., Oliveira, F., and Zimdars, D., "Thz imaging and sensing for security applications - explosives, weapons and drugs," Semiconductor Science and Technology 20(7), S266 (2005).

[23] Wang, C.-L. and Pan, C.-L., "Tunable multiterahertz beat signal generation from a two-wavelength laserdiode array," Optics letters 20(11), 1292-1294 (1995).

[24] Brown, E. R., McIntosh, K. A., Nichols, K. B., and Dennis, C. L., "Photomixing up to 3.8 thz in lowtemperature-grown gaas," Applied Physics Letters 66(3), 285-287 (1995).

[25] Leinonen, T., Ranta, S., Laakso, A., Morozov, Y., Saarinen, M., and Pessa, M., "Dual-wavelength generation by vertical external cavity surface-emitting laser," Optics express 15(20), 13451-13456 (2007).

[26] Pal, V., Trofimoff, P., Miranda, B.-X., Baili, G., Alouini, M., Morvan, L., Dolfi, D., Goldfarb, F., Sagnes, I., Ghosh, R., et al., "Measurement of the coupling constant in a two-frequency vecsel," Optics express 18(5), 5008-5014 (2010).

[27] Chernikov, A., Wichmann, M., Shakfa, M., Scheller, M., Moloney, J., Koch, S., and Koch, M., "Timedynamics of the two-color emission from vertical-external-cavity surface-emitting lasers," Applied Physics Letters 100(4), 041114 (2012).

[28] Wichmann, M., Shakfa, M. K., Zhang, F., Heinen, B., Scheller, M., Rahimi-Iman, A., Stolz, W., Moloney, J. V., Koch, S. W., and Koch, M., "Evolution of multi-mode operation in vertical-external-cavity surfaceemitting lasers," Optics Express 21(26), 31940-31950 (2013).

[29] Wichmann, M., Town, G., Quante, J., Gaafar, M., Rahimi-Iman, A., Stolz, W., Koch, S., and Koch, M., "Antiphase noise dynamics in a dual-wavelength vertical-external-cavity surface-emitting laser," IEEE Photonics Technology Letters 27(19), 2039-2042 (2015).

[30] Scheller, M., Baker, C. W., Koch, S. W., Moloney, J. V., and Jones, R. J., "High power dual-wavelength vecsel based on a multiple folded cavity," IEEE Photonics Technology Letters 29(10), 790-793 (2017).

[31] De, S., Pal, V., El Amili, A., Pillet, G., Baili, G., Alouini, M., Sagnes, I., Ghosh, R., and Bretenaker, F., "Intensity noise correlations in a two-frequency vecsel," Optics express 21(3), 2538-2550 (2013).

[32] Koryukin, I. and Povyshev, V., "Antiphase dynamics of a multimode quantum well semiconductor laser," Laser physics 17(5), 680-683 (2007).

[33] Ahmed, M. and Yamada, M., "Influence of instantaneous mode competition on the dynamics of semiconductor lasers," IEEE Journal of Quantum Electronics 38(6), 682-693 (2002).

[34] Yacomotti, A. M., Furfaro, L., Hachair, X., Pedaci, F., Giudici, M., Tredicce, J., Javaloyes, J., Balle, S., Viktorov, E. A., and Mandel, P., "Dynamics of multimode semiconductor lasers," Physical Review A 69(5), 053816 (2004).

[35] Matus, M., Kolesik, M., Moloney, J. V., Hofmann, M., and Koch, S. W., "Dynamics of two-color laser systems with spectrally filtered feedback," JOSA B 21(10), 1758-1771 (2004).

[36] Bäumner, A., Koch, S. W., and Moloney, J. V., "Non-equilibrium analysis of the two-color operation in semiconductor quantum-well lasers," physica status solidi (b) 248(4), 843-846 (2011).

[37] Haug, H. and Koch, S. W., [Quantum Theory of the Optical and Electronic Properties of Semiconductors], World Scientific, $5^{\text {th }}$ ed. (2009).

[38] Hader, J., Koch, S. W., and Moloney, J. V., "Microscopic theory of gain and spontaneous emission in gainnas laser material," Solid-State Electronics 47(3), 513-521 (2003). 
[39] Kilen, I., Koch, S. W., Hader, J., and Moloney, J. V., "Non-equilibrium ultrashort pulse generation strategies in vecsels," Optica 4(4), 412-417 (2017).

[40] Kilen, I., Koch, S. W., Hader, J., and Moloney, J. V., "Vecsel design for high peak power ultrashort modelocked operation," Applied Physics Letters 112(26), 262105 (2018).

[41] Kilen, I., Hader, J., Moloney, J. V., and Koch, S. W., "Ultrafast nonequilibrium carrier dynamics in semiconductor laser mode locking," Optica 1(4), 192-197 (2014).

[42] Kilen, I., Koch, S. W., Hader, J., and Moloney, J. V., "Fully microscopic modeling of mode locking in microcavity lasers," JOSA B 33(1), 75-80 (2016).

[43] Madelung, O., von der Osten, W., and Rössler, U., "Landolt-börnstein: Numerical data and functional relationships in science and technology," New series group III: Crystal and Solid State Physics 22a, 63-117 (1987).

[44] Kuhn, T., "Density matrix theory of coherent ultrafast dynamics," in [Theory of transport properties of semiconductor nanostructures], 173-214, Springer (1998).

[45] Waldmüller, I., Intersubband Dynamics in Semiconductor Quantum Wells-Linear and Nonlinear Response of Quantum Confined Electrons, $\mathrm{PhD}$ thesis, Technische Universität Berlin (2005).

[46] Liu, Y. and Ohtsubo, J., "Dynamics and chaos stabilization of semiconductor lasers with optical feedback from an interferometer," IEEE Journal of Quantum electronics 33(7), 1163-1169 (1997). 